\title{
Single-trial-based Temporal Principal Component Analysis on Extracting Event-related Potentials of Interest for an Individual Subject
}

\author{
Guanghui Zhang ${ }^{\mathrm{a}, \mathrm{b}, *}$, Xueyan $\mathrm{Li}^{\mathrm{c}}$, Yingzhi Lu ${ }^{\mathrm{d}}$, Timo Tiihonen ${ }^{\mathrm{b}}$, Zheng \\ Chang ${ }^{\mathrm{b}}$, Fengyu Cong ${ }^{\mathrm{a}, \mathrm{b}, \mathrm{e}, \mathrm{f}}$ \\ ${ }^{a}$ School of Biomedical Engineering, Faculty of Electronic Information and Electrical \\ Engineering, Dalian University of Technology, Dalian, 116024, China \\ ${ }^{b}$ Faculty of Information Technology, University of Jyväskylä, Jyväskylä,40014, Finland \\ ${ }^{c}$ School of Foreign Languages, Dalian University of Technology, Dalian, 116024, China \\ ${ }^{d}$ School of Psychology, Shanghai University of Sport, Shanghai, 200438, China. \\ ${ }^{e}$ School of Artificial Intelligence, Faculty of Electronic Information and Electrical \\ Engineering, Dalian University of Technology, Dalian,116024, China. \\ ${ }^{f}$ Key Laboratory of Integrated Circuit and Biomedical Electronic System, Dalian University \\ of Technology, Dalian, 116024, China.
}

\begin{abstract}
Temporal principal component analysis (t-PCA) has been widely used to extract event-related potentials (ERPs) at the group level of multiple subjects' ERP data. The key assumption of group t-PCA analysis is that desired ERPs of all subjects share the same waveforms (i.e., temporal components), whereas waveforms of different subjects' ERPs can be variant in phases, peak latencies and so on, to some extent. Additionally, several PCA-extracted components coming from the same ERP dataset failed to be statistically analysed simultaneously because their polarities and amplitudes were indeterminate. To fill these gaps, a novel technique was proposed and employed to extract desired ERP from single-trial EEG dataset of an individual subject. Firstly, the dataset of all trials and all conditions of one subject were stacked across electrodes to form a matrix. Secondly, the temporal and spatial PCA-components were extracted from single-trial EEG dataset matrix for each subject by t-PCA and Promax
\end{abstract}

\footnotetext{
* Corresponding author

Email addresses: zhang.guanghui@foxmail.com (Guanghui Zhang), lixueyan@dlut.edu.cn (Xueyan Li), yingzhi.lu.sus@gmail.com (Yingzhi Lu), timo.tiihonen@jyu.fi (Timo Tiihonen), zheng.chang@jyu.fi (Zheng Chang), cong@dlut.edu.cn (Fengyu Cong)
} 
rotation. Thirdly, the desired components were selected and projected to the electrode fields simultaneously to correct their variance and polarity indeterminacies. Next, single-trial EEG datasets of the back-projection were averaged to generate the waveforms of desired ERP for each subject and then amplitudes of the desired ERP were quantified. The yields indicated that the proposed approach can efficient exploit the temporal and spatial information of single-trial EEG data and can temporally filter the data to extract the desired ERPs for an individual subject.

Keywords: Event-related potentials, principal component analysis, single-trial analysis, back-projection, individual subject

\section{Introduction}

Event-related potentials (ERPs) have been widely used to investigate brain cognitive processes in many fields, such as language, neuroscience, physiology, psychology, and so forth Luck (2014). Traditionally, after pre-processed EEG data are averaged over trials under same experimental condition, amplitudes/latencies of ERPs are measured within a predefined time-window at some specific electrodes Luck (2014); Handy (2005). This traditional technique may fail to completely explore the properties of ERPs when the analysed ERPs are mixed with others in time and space domains to some degree. To fill this gap, temporal principal component analysis (PCA) has been developed and frequently used in previous ERP studies (Barry et al., 2018; Chandregowda et al. 2020, Mulligan et al., 2020; Widmann et al., 2018; Hsu et al., 2014). There are two strategies for extracting ERPs of interest when performing PCA on ERP signals for group analysis of all subjects' datasets. For the first strategy, the pre-processed single-trial EEG data are first averaged under the same condition and then the averaged datasets of all subjects are concatenated across electrodes to form a matrix. Sequentially, PCA is performed on the matrix to extract components associated with ERPs of interest Fogarty et al. (2020); Male \& Gouldthorp (2020); Severo et al. (2020); Yin et al. (2018); Bonmassar et al. 
(2020). In other words, this strategy is group PCA analysis for the averaged ERP datasets ('aPCA'). For the other strategy, single-trial EEG datasets of all subjects are stacked along electrodes to generate a matrix rather than to be averaged initially (i.e., 'sPCA') Rushby \& Barry (2009); MacDonald \& Barry (2017); MacDonald et al. (2015); Rushby et al. (2005).

Both of group PCA analysis strategies follow the same model: blind source separation (BSS) Comon \& Jutten (2010). In this study, we take the decomposition of spatial-concatenation matrix $\mathbf{Z}$ as an example. The matrix $\mathbf{Z}$ can be mathematically expressed as below Zhang et al. (2020):

$$
\mathbf{Z}=\mathbf{a}_{m, 1} \cdot \mathbf{s}_{1}(t)+\cdots+\mathbf{a}_{m, r} \cdot \mathbf{s}_{r}(t)+\cdots+\mathbf{a}_{m, R} \cdot \mathbf{s}_{R}(t)=\mathbf{A} \mathbf{S},
$$

where, $\mathbf{Z}$ is generated from either trial-averaged or single-trial datasets of all subjects; $s_{r}(t)$ represents stimulus-locked, spontaneous, or noise sources; $a_{m, r}$ is coefficient between $m^{\text {th }}$ electrode and $r^{\text {th }}$ source. The key assumption in the above equation is that all subjects' ERPs share the same temporal components (i.e., waveforms), that is to say, the waveforms of ERPs for different subjects are the same. However, even if EEG datasets of different subjects are collected under the same experimental environment, it is impossible to ensure that the waveforms of ERPs for all subjects have the same trends. Furthermore, an ERP of interest may be decomposed into several components by PCA because the latencies/phases of ERP for different subjects vary from several to dozen of milliseconds, especially for some late ERPs, such as P3, N400, and so on. Since the variances and polarities of PCA-extracted components may be different from each other, it is difficult to analyse several PCA-extracted components simultaneously in the existing toolboxes, for example, in Dien's PCA toolbox (Dien, 2010a).

Regarding the first problem, namely, the unreasonable assumption in current application of PCA that ERPs of all subjects have the same temporal components (Dien, 2012), we perform PCA on the spatial-concatenation matrix generated from each subject to extract ERPs of interest rather than from all subjects simultaneously. Therefore, for each subject, it is much reasonable to 
consider that ERPs of all trials have the same temporal components. In terms of the other problem that different PCA-extracted components cannot be statistically analysed simultaneously, we introduce back-projection theory in BSS into this study, which has been widely used in other BSS for EEG analysis, for instance, independent components analysis (ICA) Cong et al. (2011a b); Makeig et al. (1997, 1999). In practice, the back-projection is to calculate the sums of the outer-product between temporal and spatial PCA-components for reconstructing the waveforms of desired ERPs. As shown in our recent study Zhang et al. (2020), PCA is indeed a BSS approach when the sources are assumed to be correlated with each other and the back projection theory also works for PCA like that for ICA.

In order to extract ERPs of interest from actual EEG data of individual subject, five steps were involved as below (see Figure. 1). Firstly, the datasets of all trials were stacked along electrodes (i.e., spatial) to form a matrix for pre-processed EEG of an individual subject. Secondly, temporal PCA and Promax rotation were employed to decompose the matrices for different individuals separately. Next, the PCA-extracted components associated with ERPs of interest for each subject were chosen and projected to the electrode fields so that the polarity and amplitude indeterminacies of those selected components can be corrected (i.e., in microvolts). After that, the back projections of single trials were averaged separately under each experimental condition for each subject. Finally, the mean/peak amplitudes of the desired ERPs were computed within a predefined time-window at some electrodes.

Conventional time-domain analysis, aPCA, and sPCA were considered as comparison techniques and they were also applied to the analysis of the same pre-processed EEG datasets. The pre-processed EEG datasets and codes used in this study are available from: http://zhangg.net/publications/. 


\section{Data description and method}

In this section, we first briefly describe an emotional EEG dataset and then the model and procedures of PCA are introduced. Finally, how to use the proposed technique to extract and identify ERPs of interest is detailed as well.

\subsection{EEG description}

The EEG data were recorded from an emotional ERP experiment with two factors: valence (extreme, moderate, and neutral) $\times$ negative category (disgusting and fearful) Lu et al. (2016). It should be noted that two stimuli for the neutral condition (i.e., neural disgusting and neural fearful) were merged to one in previous study Lu et al. (2016). 22 subjects participated in this experiment and 19 electrodes (F3, FC3, C3, CP3, P3, Fz, FCz, Cz, CPz, Pz, F4, FC4, C4, CP4, P4, TP9, TP10, VEOG, and HEOG) were used to record EEG based on the international $10-20$ system with $1000 \mathrm{~Hz}$ sampling rate. EEG recordings were referenced at FCz (Brain Products GmbH, Germany). All impedances were less than $5 \mathrm{k} \Omega$ for each sensor of each subject. The collected EEG data were pre-processed offline. Firstly, the left and right mastoids were set to offline references. Secondly, EEG data were filtered by an infinite impulse response (IIR) band-pass filter: lower cut-off was $0.1 \mathrm{~Hz}$, higher cut-off was $30 \mathrm{~Hz}$, and slope: $48 \mathrm{~dB} /$ oct. Next, the filtered EEG data were segmented from $200 \mathrm{~ms}$ before stimulus onset to $900 \mathrm{~ms}$ after stimulus onset. Those epochs' datasets whose magnitudes exceeded $\pm 80 \mu \mathrm{V}$ were discarded and the remaining epochs were baseline corrected. Finally, EEG data of all epochs were filtered by wavelet filter Cong et al. (2015); Zhang et al. (2020) to improve signal-to-noise ratio (SNR). The parameters were set for wavelet filter as below: the number of decomposition level was 10; the select mother wavelet was 'rbio6.8'; the detail coefficients at levels 5, 6, 7, 8, and 9 were used for signal reconstruction.

The details for data collection and experiment can be found in Lu et al. (2016). 


\subsection{Mathematical model and procedures for principal component analysis}

In this subsection, we introduce the model for PCA and procedures for the application of PCA to ERP/EEG datasets.

\subsubsection{Mathematical model for principal component analysis}

The application of PCA to a spatial-stacked matrix $\mathbf{Z}$ (the rows are time points and the channels from each condition/subject/trial (optional) are in columns) obtained from EEG dataset of either single subject or multiple subjects can be illustrated by Eq. 1 Zhang et al. (2020). In Eq. 1, the number of observed signals is larger than that of sources, i.e., it is an over-determined model, and we usually change this over-determined model to determined model by some methods, for example, the accumulative explained variance Zhang et al. (2020).

$$
\mathbf{X}=\mathbf{V}^{T} \mathbf{Z}=\mathbf{V}^{\mathbf{T}} \mathbf{A} \mathbf{S}=\mathbf{H S}
$$

where $\mathbf{V}^{T}$ is the dimension reduction matrix obtained by applying some PCA algorithms to matrix $\mathbf{Z}^{T}$ and $\mathbf{H}$ is a mixing matrix.

In applications of PCA, we seek an un-mixing matrix $\mathbf{W}$ by using some rotation algorithms, such as Promax, Varimax rotations, and so forth Richman (1986); Hendrickson \& White (1964); Kaiser (1958). Once the un-mixing matrix is generated, its inverse matrix $\mathbf{B}=\mathbf{W}^{-1}$ can be obtained to estimate $\mathbf{H}$. And we can also use the unmixing matrix $\mathbf{W}$ to convert $\mathbf{X}$ into an estimated component matrix:

$$
\mathbf{Y}=\mathbf{W X}=\mathbf{W H S}
$$

here, each row of the estimate matrix $\mathbf{Y}$ represents topography of each source (i.e., rotated factor scores); $\mathbf{C}=\mathbf{W H}$ is a global matrix.

Generally, we need to choose several components derived from $\mathbf{X}$ for further analysis. However, the selected components cannot be analysed simultaneously because the polarities and amplitudes of these components are indeterminate Comon \& Jutten (2010). To solve this problem, we introduce the theory of 
back-projection into this study, which has been widely used in the applications of ICA Cong et al. (2011a b); Makeig et al. (1997, 1999). In the matrix-vector form, the back-projection is the outer-product of $k^{\text {th }}$ column of $\mathbf{B}$ with $k^{\text {th }}$ row of estimated component matrix $\mathbf{Y}$ :

$$
\mathbf{Q}_{k}=\mathbf{b}_{k} \circ \mathbf{y}_{k}
$$

where $\mathbf{Q}_{k}$ represents the back-projected signals at all the electrodes for $k^{\text {th }}$ selected component; 'o' denotes the outer product of two vectors.

Under global optimization, only one nonzero element exists in each row and each column of matrix $\mathbf{C}$ so that the extracted $k^{\text {th }}$ component can uniquely represent $j^{\text {th }}$ unknown scaled source Cong et al. (2011a b):

$$
\mathbf{Q}_{k}=\mathbf{b}_{k} \circ \mathbf{y}_{k}=\mathbf{h}_{j} \circ \mathbf{s}_{j} .
$$

Regarding the original over-determined model in Eq. 1. $k^{\text {th }}$ component generated from $\mathbf{Z}$ is selected to project back to electrode fields and this procedure can be described as below:

$$
\begin{gathered}
\hat{\mathbf{Z}}_{k}=\mathbf{u}_{k} \circ \mathbf{y}_{k}, \\
\mathbf{U}=\mathbf{V B},
\end{gathered}
$$

where $\mathbf{U}$ is the estimation of $\mathbf{A}$ and its each column demotes time course for $k^{\text {th }}$ component (i.e., the $k^{\text {th }}$ rotated factor loading).

In the application of PCA, a desired ERP is often decomposed into several components because the latencies of that ERP varied among different subjects. Therefore, those components need to be projected back to the electrode fields simultaneously based on the following rule Zhang et al. (2020):

$$
\begin{aligned}
\hat{\mathbf{Z}} & =\left[\mathbf{u}_{k_{1}}, \cdots, \mathbf{u}_{k_{r}}\right]\left[\mathbf{y}_{k_{1}}, \cdots, \mathbf{y}_{k_{r}}\right]^{T} \\
& =\mathbf{u}_{k_{1}} \circ \mathbf{y}_{k_{1}}+\cdots+\mathbf{u}_{k_{r}} \circ \mathbf{y}_{k_{r}},
\end{aligned}
$$

where $k_{1}, \cdots, k_{r}\left(1 \leq k_{r}<R\right)$ represent the orders of the identified components. 


\subsubsection{Principal component analysis procedures}

To better extract ERPs of interest from original multi-condition and multisubject ERP/EEG datasets by using PCA and Promax rotation for further analysis, the following steps are involved: arrange pre-processed ERP/EEG datasets into a matrix, estimate the number of sources, select an optimal rotation method, identify PCA-extracted-components of interest, and project the identified components to electrode fields (i.e., in microvolts).

The first step is to arrange ERP/EEG datasets into a matrix. The recorded EEG datasets are born with space and time dimensions, and thus, for multicondition and multi-subject datasets, two types of matrices can be organized Dien (2012); Dien et al. (2007, 2005). For the first type of matrix, it is formed by concatenating multi-condition and multi-subject datasets over channels, that is, time points are variables and the combinations of channels and subject-condition are observations. The related PCA procedure is named as 'temporal-PCA'. For the second type of matrix, channels are variables and the other variances (i.e., time points and subject-condition) are merged into rows as observations. Likewise, we call the performance of PCA on this type of matrix as 'spatialPCA'. In this study, the former type of matrix is used based on the following reasons. For one thing, regarding the performance of temporal- and spatialPCA, temporal-PCA can yield overall better results than spatial-PCA Dien (1998, 2012). For another thing, recorded from low-density-electrode, the data leading to the desired ERP is easily mixed with others in space domain due to the volume conduction. Thus, the ERPs of interest are much more easily to be separated from the original mixed datasets using temporal-PCA.

The second step is to estimate the number of sources. The purpose of this step is to transform the over-determined model in Eq. 1 into the determined model in Eq. 2 Several approaches have been developed to achieve this goal, such as Parallel Test Horn (1965) (which is implemented in PCA Toolkit Dien (2010a)), gap measure Cong et al. (2013), and cumulative explained variance Zhang et al. (2020); Huster \& Raud (2018); Arbel et al. (2013). Here, we 
use the last approach to estimate the number of sources and this approach is to calculate the percentage between the sums of first $R$ lambda values (one lambda corresponds to one component) over the sums of all lambda values:

$$
L=\frac{\sum_{r=1}^{R} \lambda_{r}}{\sum_{n=1}^{N} \lambda_{n}} \times 100 \%,
$$

where $R$ is the number of estimated sources; $N$ is the number of variables/time points $(N \geq R)$; The lambda values are in descending order: $\lambda_{1} \geq \cdots \geq \lambda_{n}=$ $\cdots=\lambda_{N}=\sigma^{2}$. Once $L$ is given with a specific value, for example, $95 \%, 99 \%$, and so on, the corresponded number of sources can be then obtained.

The third step is to select an optimal rotation method. The goal of rotation is to rearrange the components into simple and interpretable structures so that one component corresponds to one ERP Dien (2012). Some rotation methods, such as Promax, Varimax, and Infomax, can be utilized. The results of actual and stimulated ERP datasets indicated that Promax rotation showed more accurate results than Varimax rotation Dien et al. (2005); Dien (1998); Dien et al. (2003). And, simulation ERP studies revealed that Promax generated overall better results for temporal PCA and Infomax yields better separation for spatial PCA Dien et al. (2007); Dien (2010b).

The fourth step is to identify components of interest. Although some preprocessing steps are utilized to improve signal to noise ratio of ERP/EEG signals, that is, remove some artefacts, like DC offset, slow drift of sensors, eye movement, line noise, and muscle contraction de Cheveigné \& Nelken (2019); de Cheveigné (2020); Sai et al. (2017), the PCA-extracted components still contain spontaneous EEG brain activities, components of non-interest, components of interest, and noise activities. Therefore, we need to identify those components associated with ERPs of interest for further analysis. Generally, the identification of desired components is based on the two aspects Barry et al. (2020); Zhang et al. (2020): (1) the polarity and latency of temporal component; (2) the polarity and topographical distribution of spatial component. For example, $\mathrm{N} 2$ is the desired ERP in this study, the time-window of interest is from $190 \mathrm{~ms}$ to $290 \mathrm{~ms}$ and it is primarily found over frontocentral scalp sites. As shown in 
Figure 2, for the second PCA-extracted temporal component, the polarity is positive and peak time is $248 \mathrm{~ms}$, and active area of the corresponded spatial component is located in frontocentral sites and the polarities of these sites are negative. This indicates that this component is derived from N2 and then it is selected for next procedure.

The fifth step is to project the identified components back to the electrode fields (i.e., rescale them to microvolts). When performing PCA on an ERP dataset of multiple subjects, an ERP of interest may be decomposed into several components due to the differences are found in latencies or phases of this ERP across different subjects to some degree. The scales (polarity and variance) of those components may be variant so that those components cannot be simultaneously analysed, like in PCA Toolkit Dien (2010a). To tackle this problem, we introduce back-project theory into this study, which allows different components to analyse together and their polarity and variance indeterminacies can be also corrected.

\subsection{Proposed technique}

In order to better identify the component(s) extracted from pre-processed EEG dataset of single subject by utilizing temporal-PCA and Promax rotation, a time-window with 190-290ms is set. Meanwhile, the grand averaged topographies for different experimental conditions are calculated as template (obtained by mean measurement). In the following, Pearson's linear correlation coefficients $(\mathrm{CCs})$ between the template and trial-averaged spatial component $\underset{a v}{\stackrel{(i)}{y}} y_{r}$ are also computed separately and we set threshold to help to identify the components of interest. Here, in order to more components associated with N2 are selected and in order that more subjects are involved in further analysis, this threshold is set to 0.7. In detail, only when CCs between the PCA-extracted spatial components and the template are larger than this threshold, the corresponded components are considered for further analysis.

The following steps are taken when using proposed approach (here, we name it as 'iPCA') to extract N2 from the pre-processed EEG dataset for each subject. 
(1) Single-trial EEG dataset of $i^{\text {th }}$ subject is arranged into a matrix $\mathbf{Z}^{(i)}$ with size of $T \times\left(C \times\left(E_{1}+\cdots+E_{J}\right)\right), T$ is the number of time points, $C$ is the number of channels, and $E_{j}$ is the number of trials for $j$ condition $(j=1, \cdots, J)$.

(2) T components are extracted by performing PCA on the matrix $\left(\mathbf{Z}^{(i)}\right)^{T}$ and then $R^{(i)}$ components are retained and rotated in Matlab environment (Version 2018b, the Mathworks, Inc., Natick, MA; functions: pca.m and rotatefactors.m; $\left.R^{(i)} \leq T\right)$; Here, the number of retained components is determined based on cumulative explained variance in Eq. 9 and $\mathrm{L}$ is set to $99 \%$.

(3) Correlation coefficient between template and rth trial-averaged spatial component $\stackrel{(i)}{a v} \mathbf{y}_{r}$ is computed.

(4) These components associated with N2 are identified for the next procedure according to three aspects as below. Firstly, whether CC value between the template and $r^{t h}$ trial-averaged spatial component $\underset{a v}{(i)} \mathbf{y}_{r}$ is more than 0.7; Secondly, whether the latency of $r^{t h}$ temporal component $\mathbf{u}_{r}^{(i)}$ fell in the predefined time window (190-290ms); Thirdly, whether polarities between temporal and spatial components are different, for example, polarity of temporal component is negative and the values in frontocentral sites should be below zero for spatial component in this study.

(5) Project the identified components to electrode fields for correcting their variance and polarity indeterminacies and reconstruct a new matrix $\mathbf{Z}^{(i)}=$ $\mathbf{u}_{k_{1}}^{(i)} \circ \mathbf{y}_{k_{1}}^{(i)}+\cdots+\mathbf{u}_{k_{r}}^{(i)} \circ \mathbf{y}_{k_{r}}^{(i)}$.

(6) Repeat steps (1)-(5) until the EEG datasets of all subjects are decomposed.

\section{Data analysis and results}

Two subjects were not involved in final analysis based on the steps in Section 2.3 One subject was discarded because about $37 \%$ trials were removed out during the pre-processing steps. For the other subject, latencies of three PCAextracted temporal components, i.e., $243 \mathrm{~ms}, 194 \mathrm{~ms}$, and $286 \mathrm{~ms}$, were located in the predefined time-window (190-290ms), but all the correlation coefficients (i.e., 
$0.22,0.29$, and 0.16 ) between template and the corresponded spatial components were smaller than the pre-set threshold (i.e., 0.7).

The mean/peak amplitudes of N2 were quantified with the proposed and the other three comparison techniques separately. In detail, in terms of the first comparison technique (i.e., 'WF'), in order to compare the differences of amplitudes for $\mathrm{N} 2$ obtained by using conventional time-domain analysis among different experimental conditions for the wavelet filtered datasets, EEG datasets of all remained trials were first averaged under same experimental condition for each subject. Then, mean/peak amplitudes of N2 were separately measured within 190-290ms at FC3, FCz, FC4, C3, Cz, and C4 electrodes. Finally, we used two-way repeated measurement ANOVA with valence (extreme, moderate, and neutral) $\times$ negative-category (disgusting and fearful) as within-subject factors to compute the statistical results for the measured peak/mean amplitudes separately. Greenhous-Geisser method was used for correcting the number of degrees of freedom.

To better identify PCA-extracted components of interest obtained by second and third comparison techniques, we also defined a time-window from 190$290 \mathrm{~ms}$ and only those components, whose latencies were located in the defined time-window, were considered for the next procedure. Moreover, CCs between spatial component and template (i.e., the grand averaged topographies of N2 obtained by using mean measurement for the first comparison technique) were also calculated. As for the second comparison technique (i.e., 'aPCA'), the original sing-trial datasets were first averaged under each condition for each subject separately. Secondly, the averaged datasets were spatial-stacked to generate a matrix and temporal-PCA is performed on this matrix to decomposition into 1100 components (i.e., the number of time points). Next, 15 components were retained which accounted for $99 \%$ of total variance and they were then rotated by Promax rotation. The 2nd (explained $8.6 \%$ of variance, CC: 1.000$), 4$ th (explained $2.5 \%$ of variance, CC: 0.990 ), and 9 th (explained $0.42 \%$ of variance, CC: 0.985 ) were identified for back-projection to rebuild a new matrix (see Figure 2). Apart from the first step as mentioned in the first comparison technique, the 
remained steps were also employed to measure the mean and peak amplitudes of N2.

Regarding the third comparison technique (i.e., 'sPCA'), the pre-processed EEG of all trials were first concatenated over channels to form a matrix. Secondly, 31 components were reserved and rotated by temporal-PCA and Promax rotation, which also accounted for $99 \%$ of total variance. The 3rd (explained $7.55 \%$ of variance, CC: 1.000 ), 10th (explained $1.97 \%$ of variance, CC: 0.989 ), and 12 th (explained $1.57 \%$ of variance, CC: 0.988 ) were determined for backprojection (see Figure 3). Likewise, to measure the statistical analysis results of mean/peak amplitudes for N2, the steps, which were used in the first comparison technique, were applied to the back-projection as well.

Similarly, for the proposed technique (i.e., ' $\mathrm{PCA}$ '), the procedures as described in Section 2.3 were first employed to extract components related to N2. Secondly, the steps as applied in the first comparison technique were also carried out for the back-projection of individual subject. The specific retained and identified components for different subjects could be found in Table 1 .

Additionally, aim at assessing the stability/consistency of N2 among different subjects, the correlation coefficients between topographies of any two different subjects were also computed, herein, we called the correlation coefficient yields as 'similarities' to avoid confusion with the correlation coefficients between template with PCA-extracted spatial components.

To assess the performances of different techniques, the paired t-test was used to identify the differences of similarities between the proposed technique and any one of other techniques.

Figure 4 displays the grand averaged waveforms and topographies of different experimental conditions for four different techniques: WF (Upper panel: red solid line), iPCA (Upper panel: black dotted line), aPCA (Middle panel: blue solid line), and sPCA (Lower panel: green solid line). We observed that the reconstructed waveforms and topographies of N2 under different experimental conditions were highly similar to each other when using three different PCA techniques. 
Figure 5 represents the mean/peak amplitudes of individual subjects under each condition obtained by different techniques. The mean/peak amplitudes for all individual subjects obtained by the proposed technique were smaller than 0 and this indicated that waveforms of $\mathrm{N} 2$ were well estimated by PCA and Promax rotation for each subject, but not for the other comparison techniques. Moreover, the standard values of proposed techniques under different experimental conditions were smaller than those of other techniques using both mean and peak measurements.

The statistical results of mean/peak amplitudes for N2 obtained by four techniques were computed separately. For the mean measurement, the statistical results of mean amplitudes for $\mathrm{N} 2$ revealed that the main effect of valence was significant for different techniques separately (WF: $\mathrm{F}(2,38)=30.914, \eta_{p}^{2}=0.619$, $\mathrm{p}<0.0001 ;$ iPCA: $\mathrm{F}(2,38)=25.957, \eta_{p}^{2}=0.577, \mathrm{p}<0.0001 ;$ aPCA: $\mathrm{F}(2,38)$ $=30.433, \eta_{p}^{2}=0.616, \mathrm{p}<0.0001 ; \mathrm{sPCA}: \mathrm{F}(2,38)=30.466, \eta_{p}^{2}=0.616, \mathrm{p}<$ 0.0001). Likewise, a significant interaction effect between valence and negativecategory was also found for $\mathrm{WF}\left(\mathrm{F}(2,38)=5.763, \eta_{p}^{2}=0.233, \mathrm{p}=0.007\right)$, iPCA $\left(\mathrm{F}(2,38)=5.291, \eta_{p}^{2}=0.218, \mathrm{p}=0.010\right), \mathrm{aPCA}\left(\mathrm{F}(2,38)=5.852, \eta_{p}^{2}=0.235\right.$, $\mathrm{p}=0.006)$, and $\mathrm{sPCA}\left(\mathrm{F}(2,38)=5.877, \eta_{p}^{2}=0.236, \mathrm{p}=0.006\right)$. However, main effect of negative-category did not reach significant level for $\operatorname{WF}(\mathrm{F}(1,19)$ $\left.=0.649, \eta_{p}^{2}=0.033, \mathrm{p}=0.431\right), \operatorname{iPCA}\left(\mathrm{F}(1,19)=0.643, \eta_{p}^{2}=0.033, \mathrm{p}=0.432\right)$, $\operatorname{aPCA}\left(\mathrm{F}(1,19)=0.696, \eta_{p}^{2}=0.035, \mathrm{p}=0.414\right)$, and $\operatorname{sPCA}(\mathrm{F}(1,19)=0.739$, $\left.\eta_{p}^{2}=0.037, \mathrm{p}=0.401\right)$. Regarding the statistical results of peak amplitudes for N2, the main effect of negative-category was also insignificant. Similarly, we found that the interaction effect between valence and negative-category did not reach significant level when using $\mathrm{WF}\left(\mathrm{F}(2,38)=1.731, \eta_{p}^{2}=0.084, \mathrm{p}=0.194\right)$, $\operatorname{aPCA}\left(\mathrm{F}(2,38)=2.771, \eta_{p}^{2}=0.127, \mathrm{p}=0.077\right)$, and $\operatorname{sPCA}(\mathrm{F}(2,38)=2.062$, $\left.\eta_{p}^{2}=0.098, \mathrm{p}=0.144\right)$ techniques. By contrast, the yields of iPCA indicated that a significant interaction effect between valence and negative-category was observed $\left(\mathrm{F}(2,38)=4.794, \eta_{p}^{2}=0.201, \mathrm{p}=0.014\right)$.

For the yields of similarities, as displayed in Figure. 6 (a) and (b), the mean similarities of different conditions for the proposed technique were higher 
than those of the other three techniques, especially when using peak measurement. By contrast, the standard similarity of each condition for the proposed techniques was smaller than those of other comparison techniques. The paired t-test results of similarities indicated that significant difference under each condition could be obtained between the proposed technique and any one of other comparison techniques for both mean and peak measurements.

\section{Conclusion and discussion}

In this study, we developed a novel technique (named as 'iPCA') to extract the temporal and spatial information of desired ERPs from an individual subject dataset of an emotional experiment with two factors using temporal PCA and Promax rotation. The proposed technique can efficiently capture properties of N2 (here, peak amplitude) from individual subject so that main effects of different negative-category conditions revealed different trends under different valences, which were consistent with the previous study [42], but not for other comparison techniques. Meanwhile, the proposed technique also helped to enhance the similarities of $\mathrm{N} 2$ among different subjects. We concluded that the proposed technique produces much better yields than compared group analysis techniques as shown in Section 3.

The main difference between the proposed technique and the conventional time-domain analysis (i.e., 'WF') is that WF only uses the temporal (i.e., amplitude) property of $\mathrm{N} 2$ and the obtained results are still mixtures, but the proposed technique explores both temporal and spatial characteristics of N2. Moreover, compared with the other two group-PCA-analysis ('aPCA' and 'sPCA') techniques in which ERPs of interest are extracted from all subjects, the proposed technique explores the desired ERPs from the pre-processed dataset of each subject. Traditional group PCA analysis ('aPCA' and 'sPCA') assumed that the number and the order of sources were the same for all subjects, and as a result, ERPs of all subjects have same waveforms, but distinct in topographies Dien (2012). We expect that the waveforms of ERPs for all subjects are highly 
similar to each other, but it is impossible to fulfil this expectation in real ERP datasets. When using the proposed technique, we considered that the waveforms of all trials are the same for the PCA-extracted temporal component, which is satisfied with the assumption of actual ERP datasets. Consequently, we strongly suggest extracting the properties of ERPs of interest from single-trial dataset of individual subject separately rather than from all subjects synchronously should be adopted and also conformed to this study.

Moreover, the back-projection theory is introduced to this study which allows several PCA-extracted components to be analysed simultaneously in microvolts. We strongly expect that the elicited waveforms of a desired ERP are similar among different subjects. However, in actual ERP datasets, not only the shapes of waveforms for the desired ERP are varied among different subjects, but both latencies and phases of the desired ERP are also different. Therefore, an ERP of interest is often decomposed into several PCA-components when performing temporal PCA in the matrix obtained from pre-processed ERP/EEG datasets as shown in Figures 1 and 2. This trend is also confirmed in several previous studies Zhang et al. (2020); Li et al. (2019). In the existing toolboxes, for example, Dien's PCA Toolkit Dien (2010a) and Kayser's erpPCA Kayser \& Tenke (2003), the scales (i.e., polarity and amplitude) of these PCAextracted components are poorly considered so that these components cannot be directly analysed simultaneously when their scales are different. Although PCA-extracted components can be analysed separately and related statistical analysis results can also be provided separately in both of the toolboxes, those separately analysed results only reveal the partial information of a desired ERP. To overcome this problem, we applied the theory of back-projection, which is widely used in the applications of ICA to EEG signals Cong et al. (2011a); Makeig et al. (1997, 1999); Cong et al. (2013). Indeed, the back-projection calculates the sums of the outer-product between temporal and spatial components, as displayed in Eq. 8, so that indeterminacies of polarity and amplitude for those selected components can be corrected. As a result, not only several components derived from either the same ERP or different ERPs for all sub- 
jects can be quantified, but PCA-extracted components coming from individual subjects' datasets can also be analysed together (i.e., the proposed technique).

In addition, the similarities of topographies among different subjects are used to assess the stability/reliability of an ERP of interest and those are also used to measure the performances of different techniques. In terms of an ERP, it is characterized by both a specific time course and a specific topography. For instance, in a typical oddball paradigm, P3a can be elicited about $250-280 \mathrm{~ms}$ at frontalcentral sites Polich (2007); Comerchero \& Polich (1999). Generally, in an ERP experiment, ERPs are often quantified from tens or hundreds of subjects, and thus, we expect that brain activities related to stimulus onset can be observed from all subjects so that the related experiment is reproducible and reliable. A reliable ERP response means that higher correlation coefficients of topographies between any two different subjects can be obtained. As shown in Figure 6 (a) and (b), regarding both mean and peak measurements, the mean similarities of different experimental conditions for the proposed techniques are higher than those of the other three techniques but have lower standard similarities. Meanwhile, paired t-test results of similarities revealed that a significant difference of similarities between the proposed technique and any one of three comparison techniques can be obtained in any experimental condition (see Figure 6 (a) and (b)). These clarified that a more reliable ERP can be extracted from single subject by the proposed technique.

In summary, the results of the proposed techniques lead to the following four suggestions: (1) ERPs of interest can be efficiently extracted from single subject by PCA decomposition rather than from the datasets of all subjects simultaneously (which is often used in PCA Toolkit Dien (2010a)). (2) Although all subjects perform same experimental tasks under the same situations, not all subjects can elicit ERPs of interest. (3) Back-projection of several components corresponding to ERPs of interest can overcome the scale differences in polarity and amplitude for the PCA-extracted components so that we can study them in microvolts. (4) The similarities of topographies among different subjects can be used as both a criterion to evaluate the performance of the used techniques 
and a method to judge whether the analysed ERPs are repeatable and reliable or not.

Furthermore, there are some potential limitations and further directions. Firstly, we only perform PCA on the spatial-stacked matrix (i.e., temporalPCA) to extract N2 of interest in current study. Although the proposed technique produces much better overall results than those of all the other techniques, this conclusion is not further validated in the applications of spatial PCA. Secondly, the comparison of yields between PCA and ICA is not included in this study. Many applications of ICA indicated that ERPs of interest can also be efficiently extracted from both single-trial EEG and averaged ERP datasets Lee et al. (2016); Wessel (2018); Cong et al. (2013). It is worth investigating the information of the desired ERP using ICA from either average traces or singletrial traces. Thirdly, the identification of PCA-extracted components associated with the desired ERP seems to be a subjective way in this study, which contains two aspects. One aspect is based on the properties of extracted components in temporal and spatial domains. The other aspect is based on the correlation coefficients between template and the related spatial components (i.e., topographies). The current threshold is not further investigated for the extraction of other ERPs. Thus, in the future work, the above mentioned limitations should be overcome.

\section{Data Availability}

The data and MATLAB script codes used for supporting the findings in this study are available from the following link: http://zhangg.net/publications/.

\section{Conflicts of Interest}

None of the authors have potential conflicts of interest to be disclosed. 


\section{Acknowledgments}

This work was supported by National Natural Science Foundation of China (Grant No.91748105), National Foundation in China (No. JCKY2019110B009 \& 2020-JCJQ-JJ-252) and the Fundamental Research Funds for the Central Universities [DUT20LAB303 \& DUT20LAB308] in Dalian University of Technology in China, and the scholarships from China Scholarship Council (No.201806060165). This study is to memorize Prof. Tapani Ristaniemi for his great help to Guanghui Zhang, Fengyu Cong and the other authors.

\section{References}

Arbel, Y., Goforth, K., \& Donchin, E. (2013). The good, the bad, or the useful? the examination of the relationship between the feedback-related negativity (FRN) and long-term learning outcomes. Journal of Cognitive Neuroscience, 25, 1249-1260. doi:10.1162/jocn_a_00385.

Barry, R. J., De Blasio, F. M., \& Fogarty, J. S. (2018). A processing schema for children in the auditory equiprobable Go/NoGo task: ERP components and behaviour. International Journal of Psychophysiology, 123, 74-79. doi 10. 1016/j.ijpsycho.2017.10.014.

Barry, R. J., Steiner, G. Z., De Blasio, F. M., Fogarty, J. S., Karamacoska, D., \& MacDonald, B. (2020). Components in the P300: Don't forget the Novelty P3! Psychophysiology, 57, e13371. doi 10.1111/psyp.13371.

Bonmassar, C., Widmann, A., \& Wetzel, N. (2020). The impact of novelty and emotion on attention-related neuronal and pupil responses in children. Developmental Cognitive Neuroscience, 42, 100766. doi $10.1016 /$ j.dcn.2020. 100766 .

Chandregowda, A., Arbel, Y., \& Donchin, E. (2020). Seeking neurophysiological manifestations of speech production: An ERP study. International Journal of Psychophysiology, 147, 137-146. doi 10.1016/j.ijpsycho.2019.10.013. 
de Cheveigné, A. (2020). Zapline: A simple and effective method to remove power line artifacts. NeuroImage, 207, 116356. doi $10.1016 / \mathrm{j}$.neuroimage. 2019.116356 .

de Cheveigné, A., \& Nelken, I. (2019). Filters: when, why, and how (not) to use them. Neuron, 102, 280-293. doi $10.1016 / j$.neuron.2019.02.039.

Comerchero, M. D., \& Polich, J. (1999). P3a and p3b from typical auditory and visual stimuli. Clinical Neurophysiology, 110, 24-30. doi10.1016/ S0168-5597(98)00033-1.

Comon, P., \& Jutten, C. (2010). Handbook of Blind Source Separation: Independent component analysis and applications. Academic Press.

Cong, F., He, Z., Hämäläinen, J., Leppänen, P. H., Lyytinen, H., Cichocki, A., \& Ristaniemi, T. (2013). Validating rationale of group-level component analysis based on estimating number of sources in EEG through model order selection. Journal of Neuroscience Methods, 212, 165-172. doi $10.1016 / \mathrm{j}$. jneumeth.2012.09.029.

Cong, F., Kalyakin, I., Chang, Z., \& Ristaniemi, T. (2011a). Analysis on subtracting projection of extracted independent components from eeg recordings. Biomedical Engineering/Biomedizinische Technik, 56, 223-234. doi $10.1515 /$ bmt.2011.102.

Cong, F., Kalyakin, I., \& Ristaniemi, T. (2011b). Can back-projection fully resolve polarity indeterminacy of independent component analysis in study of event-related potential? Biomedical Signal Processing and Control, 6, 422-426. doi $10.1016 / \mathrm{j} . \mathrm{bspc} .2010 .05 .006$.

Cong, F., Ristaniemi, T., \& Lyytinen, H. (2015). Wavelet filter design based on frequency responses for filtering erp data with duration of one epoch. Advanced Signal Processing on Brain Event-Related Potentials, World Scientific, Singapore, (pp. 15-49). doi:10.1142/9789814623094_0002. 
Dien, J. (1998). Addressing misallocation of variance in principal components analysis of event-related potentials. Brain Topography, 11, 43-55. doi 10 . 1023/A:1022218503558.

Dien, J. (2010a). The ERP PCA Toolkit: An open source program for advanced statistical analysis of event-related potential data. Journal of Neuroscience Methods, 187, 138-145. doi:10.1016/j.jneumeth.2009.12.009.

Dien, J. (2010b). Evaluating two-step pca of erp data with geomin, infomax, oblimin, promax, and varimax rotations. Psychophysiology, 47, 170-183. doi: $10.1111 / \mathrm{j} .1469-8986.2009 .00885 \cdot \mathrm{x}$.

Dien, J. (2012). Applying principal components analysis to event-related potentials: a tutorial. Developmental Neuropsychology, 37, 497-517. doi $10.1080 /$ 87565641.2012 .697503 .

Dien, J., Beal, D. J., \& Berg, P. (2005). Optimizing principal components analysis of event-related potentials: matrix type, factor loading weighting, extraction, and rotations. Clinical Neurophysiology, 116, 1808-1825. doi 10 . 1016/j.clinph.2004.11.025.

Dien, J., Frishkoff, G. A., Cerbone, A., \& Tucker, D. M. (2003). Parametric analysis of event-related potentials in semantic comprehension: evidence for parallel brain mechanisms. Cognitive Brain Research, 15, 137-153. doi 10 . 1016/S0926-6410(02)00147-7.

Dien, J., Khoe, W., \& Mangun, G. R. (2007). Evaluation of PCA and ICA of simulated ERPs: Promax vs. Infomax rotations. Human Brain Mapping, 28, 742-763. doi $10.1002 / \mathrm{hbm} .20304$.

Fogarty, J. S., Barry, R. J., \& Steiner, G. Z. (2020). Auditory stimulusand response-locked ERP components and behavior. Psychophysiology, 57, e13538. doi:10.1111/psyp.13538.

Handy, T. C. (2005). Event-related potentials: A methods handbook. MIT press. 
Hendrickson, A. E., \& White, P. O. (1964). Promax: A quick method for rotation to oblique simple structure. British Journal of Statistical Psychology, 17, 65-70. doi $10.1111 /$ j.2044-8317.1964.tb00244.x.

Horn, J. L. (1965). A rationale and test for the number of factors in factor analysis. Psychometrika, 30, 179-185. doi 10.1007/BF02289447.

Hsu, Y.-F., Hamalainen, J., \& Waszak, F. (2014). Both attention and prediction are necessary for adaptive neuronal tuning in sensory processing. Frontiers in Human Neuroscience, 8, 152. doi 10.3389/fnhum.2014.00152.

Huster, R. J., \& Raud, L. (2018). A tutorial review on multi-subject decomposition of EEG. Brain Topography, 31, 3-16. doi 10.1007/s10548-017-0603-x.

Kaiser, H. F. (1958). The varimax criterion for analytic rotation in factor analysis. Psychometrika, 23, 187-200. doi 10.1007/BF02289233.

Kayser, J., \& Tenke, C. E. (2003). Optimizing PCA methodology for ERP component identification and measurement: theoretical rationale and empirical evaluation. Clinical Neurophysiology, 114, 2307-2325. doi 10.1016/ S1388-2457(03) 00241-4.

Lee, W. L., Tan, T., Falkmer, T., \& Leung, Y. H. (2016). Single-trial eventrelated potential extraction through one-unit ica-with-reference. Journal of Neural Engineering, 13, 066010. doi 10.1088/1741-2560/13/6/066010.

Li, X., Zhang, G., Zhou, C., \& Wang, X. (2019). Negative emotional state slows down movement speed: behavioral and neural evidence. PeerJ, 7, e7591. doi:10.7717/peerj.7591.

Lu, Y., Luo, Y., Lei, Y., Jaquess, K. J., Zhou, C., \& Li, H. (2016). Decomposing valence intensity effects in disgusting and fearful stimuli: an event-related potential study. Social Neuroscience, 11, 618-626. doi 10.1080/17470919. 2015.1120238. 
Luck, S. J. (2014). An introduction to the event-related potential technique. MIT press.

MacDonald, B., \& Barry, R. J. (2017). Significance and novelty effects in singletrial ERP components and autonomic responses. International Journal of Psychophysiology, 117, 48-64. doi:10.1016/j.ijpsycho.2017.03.007.

MacDonald, B., Barry, R. J., \& Bonfield, R. C. (2015). Trials and intensity effects in single-trial erp components and autonomic responses in a dishabituation paradigm with very long isis. International Journal of Psychophysiology, 98, 394-412. doi 10.1016/j.ijpsycho.2015.08.002.

Makeig, S., Jung, T.-P., Bell, A. J., Ghahremani, D., \& Sejnowski, T. J. (1997). Blind separation of auditory event-related brain responses into independent components. Proceedings of the National Academy of Sciences, 94, 1097910984. doi 10.1073/pnas.94.20.10979.

Makeig, S., Westerfield, M., Jung, T.-P., Covington, J., Townsend, J., Sejnowski, T. J., \& Courchesne, E. (1999). Functionally independent components of the late positive event-related potential during visual spatial attention. Journal of Neuroscience, 19, 2665-2680. doi 10.1523/JNEUROSCI. 19-07-02665.1999.

Male, A. G., \& Gouldthorp, B. (2020). Hemispheric differences in perceptual integration during language comprehension: An ERP study. Neuropsychologia, 139, 107353. doi $10.1016 /$ j.neuropsychologia.2020.107353

Mulligan, E. M., Infantolino, Z. P., Klein, D. N., \& Hajcak, G. (2020). Developmental trajectory of the late positive potential: Using temporal-spatial PCA to characterize within-subject developmental changes in emotional processing. Psychophysiology, 57, e13478. doi 10.1111/psyp.13478.

Polich, J. (2007). Updating p300: an integrative theory of p3a and p3b. Clinical Neurophysiology, 118, 2128-2148. doi:10.1016/j.clinph.2007.04.019. 
Richman, M. B. (1986). Rotation of principal components. Journal of Climatology, 6, 293-335. doi 10.1002/joc.3370060305.

Rushby, J. A., \& Barry, R. J. (2009). Single-trial event-related potentials to significant stimuli. International Journal of Psychophysiology, 74, 120-131. doi $10.1016 /$ j.ijpsycho.2009.08.003.

Rushby, J. A., Barry, R. J., \& Doherty, R. J. (2005). Separation of the components of the late positive complex in an erp dishabituation paradigm. Clinical Neurophysiology, 116, 2363-2380. doi 10.1016/j.clinph.2005.06.008.

Sai, C. Y., Mokhtar, N., Arof, H., Cumming, P., \& Iwahashi, M. (2017). Automated classification and removal of EEG artifacts with SVM and waveletICA. IEEE Journal of Biomedical and Health Informatics, 22, 664-670. doi:10.1109/JBHI.2017.2723420.

Severo, M. C., Paul, K., Walentowska, W., Moors, A., \& Pourtois, G. (2020). Neurophysiological evidence for evaluative feedback processing depending on goal relevance. NeuroImage, 215, 116857. doi:10.1016/j.neuroimage. 2020 . 116857.

Wessel, J. R. (2018). A neural mechanism for surprise-related interruptions of visuospatial working memory. Cerebral Cortex, 28, 199-212. doi 10.1093/ cercor/bhw367.

Widmann, A., Schröger, E., \& Wetzel, N. (2018). Emotion lies in the eye of the listener: Emotional arousal to novel sounds is reflected in the sympathetic contribution to the pupil dilation response and the P3. Biological Psychology, 133, 10-17. doi $10.1016 /$ j.biopsycho.2018.01.010.

Yin, H., Wang, Y., Zhang, X., \& Li, P. (2018). Feedback delay impaired reinforcement learning: Principal components analysis of reward positivity. Neuroscience Letters, 685, 179-184. doi:10.1016/j.neulet.2018.08.039. 
bioRxiv preprint doi: https://doi.org/10.1101/2021.03.10.434892; this version posted April 20, 2021. The copyright holder for this preprint (which

was not certified by peer review) is the author/funder, who has granted bioRxiv a license to display the preprint in perpetuity. It is made available under aCC-BY-NC 4.0 International license.

Zhang, G., Li, X., \& Cong, F. (2020). Objective extraction of evoked eventrelated oscillation from time-frequency representation of event-related potentials. Neural Plasticity, 2020. doi 10.1155/2020/8841354. 


\section{Exploring ERP from individual subject EEG using temporal-PCA}

(b)

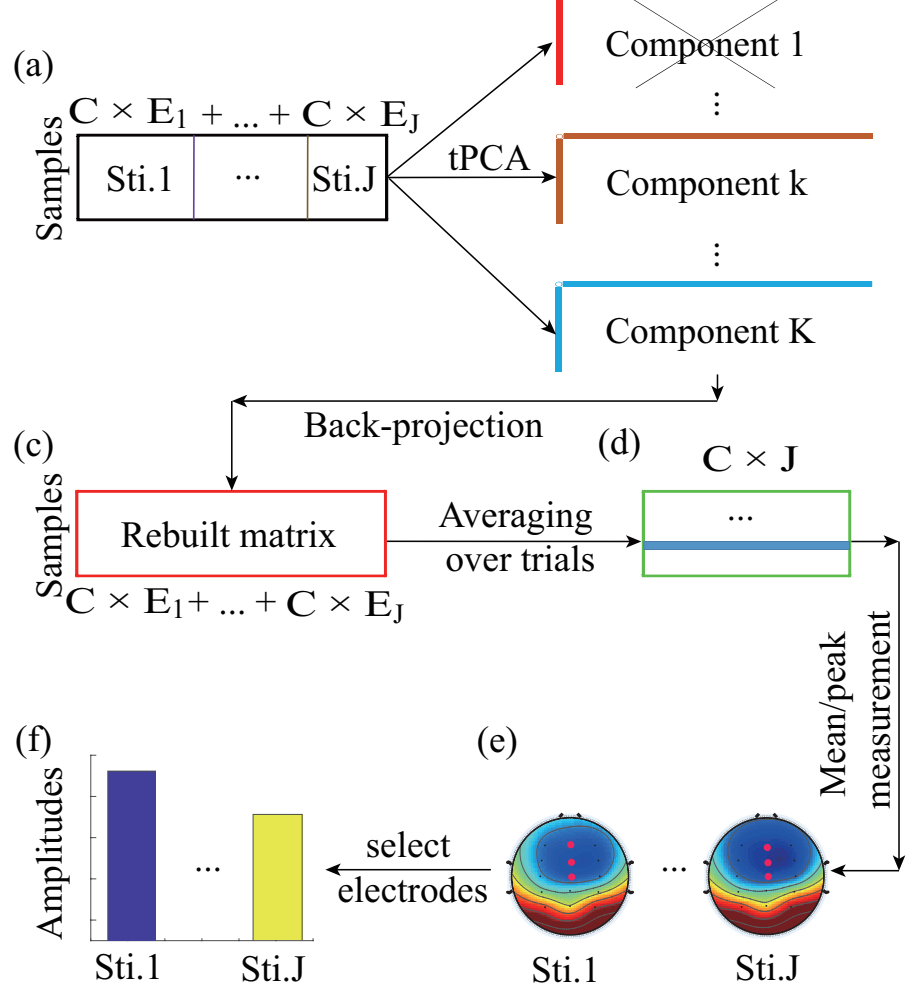

Figure 1: The illustration of the proposed technique. (a) The single-trial EEG for individual subject are arranged into a matrix over channels (trial numbers of condition $1, \ldots, J$ are $E_{1}$, $\ldots, E_{J}$, respectively; $C$ is number of electrodes). (b) The matrix are decomposed into sums of $\mathrm{R}$ components by using temporal-PCA and Promax rotation. (c) Projecting the selected components back to electrode fields for building a new matrix. (d) Waveforms of single-trial EEG are averaged under each condition. (e)-(f) Calculating the amplitudes of the desired ERP for different conditions within a defined time-window at some specific electrodes. 

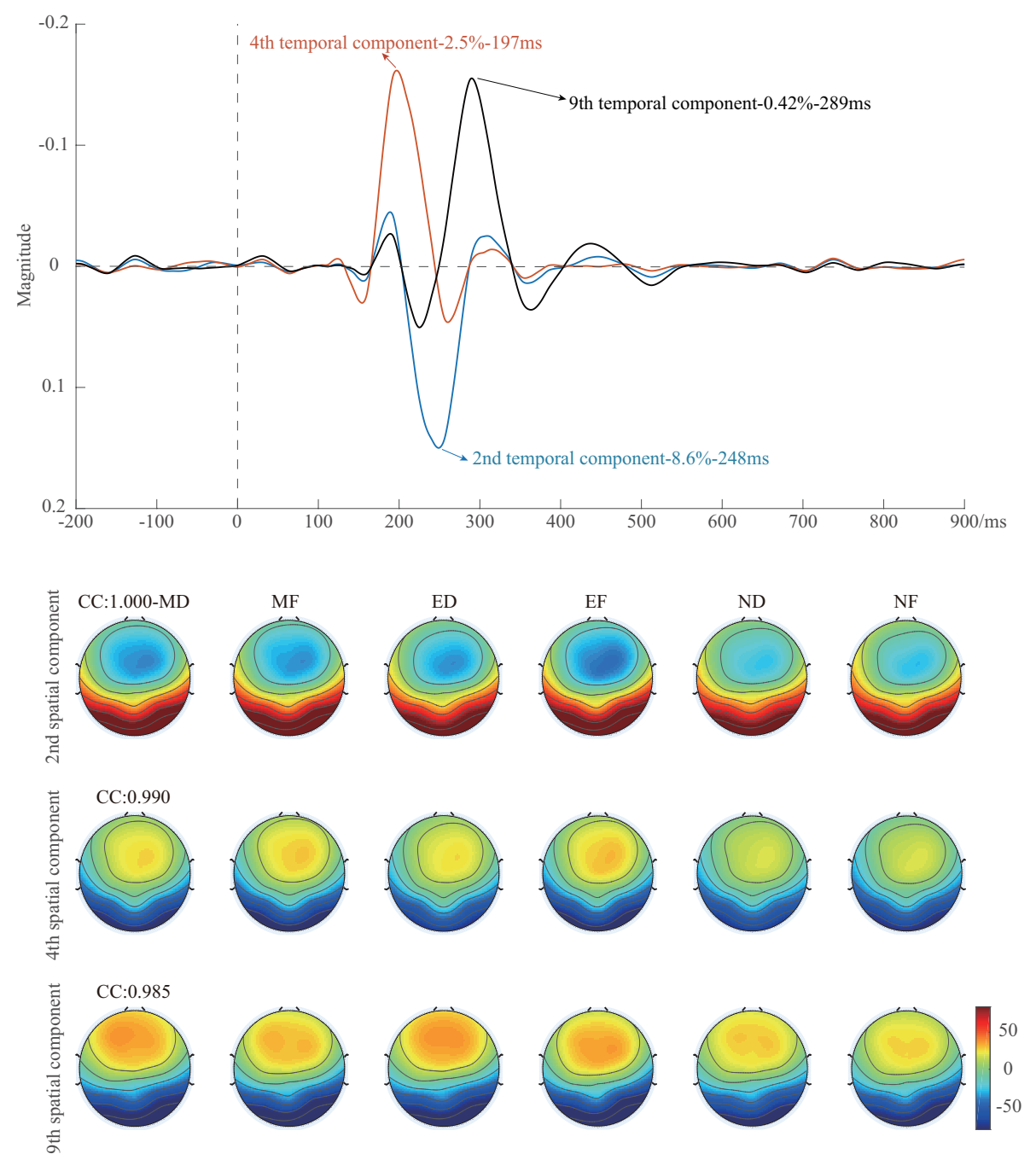

Figure 2: The time courses and topographies for three selected components when using aPCA. The percentage of variance explained by each component is also revealed and correlation coefficient (CC) between spatial component and template (i.e., topographies obtained by using conventional time domain analysis for the wavelet filtered data) is separately computed as well. MD: moderate disgusting; MF: moderate fearful; ED: extreme disgusting; EF: extreme fearful; ND: Neutral disgusting; NF: neutral fearful. 
bioRxiv preprint doi: https://doi.org/10.1101/2021.03.10.434892; this version posted April 20, 2021. The copyright holder for this preprint (which

was not certified by peer review) is the author/funder, who has granted bioRxiv a license to display the preprint in perpetuity. It is made available under aCC-BY-NC 4.0 International license.
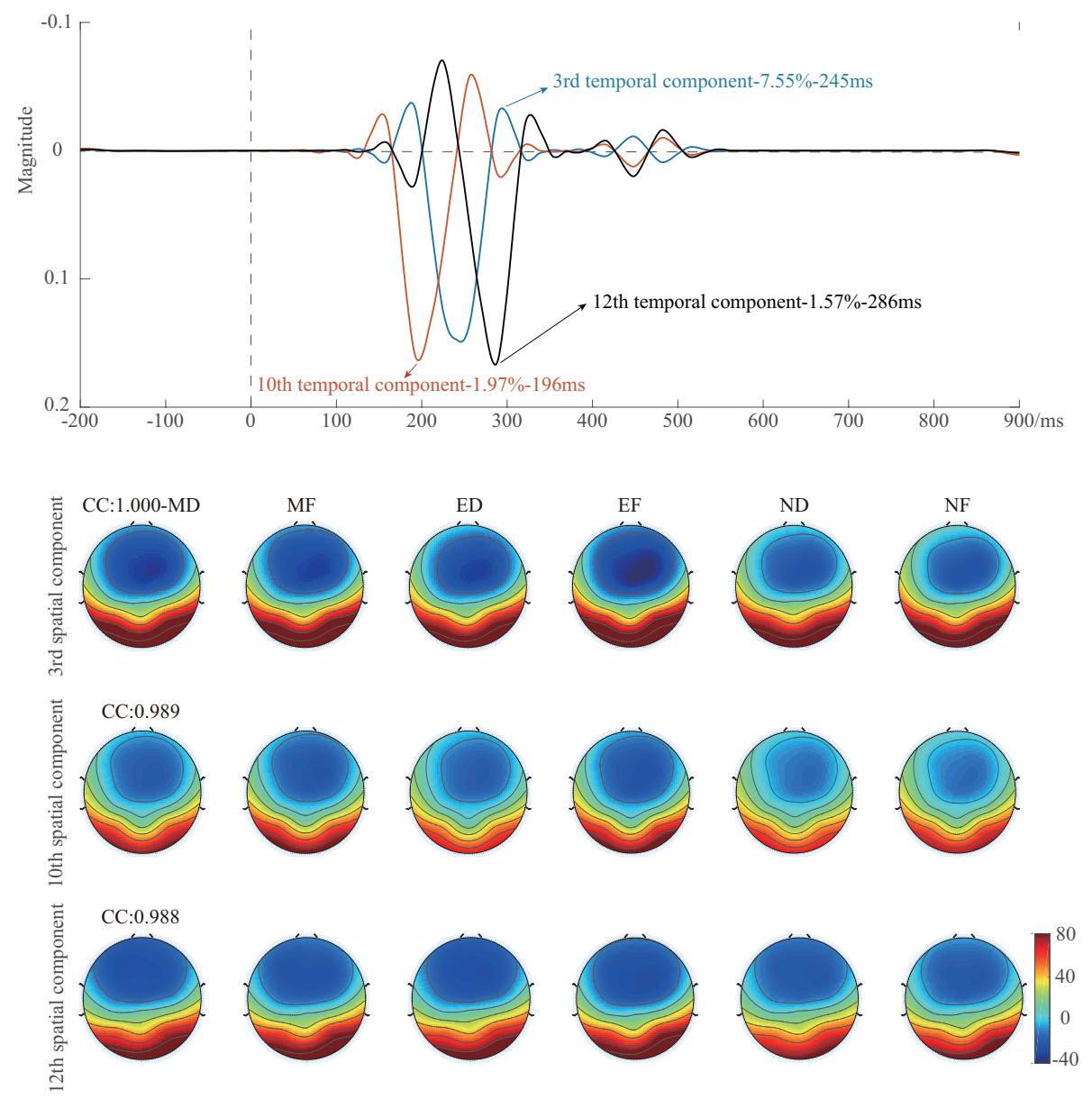

Figure 3: The time courses and topographies for three selected components when using sPCA. The percentage of variance explained by each component is also revealed and correlation coefficient (CC) between spatial component and template (i.e., topographies obtained by using conventional time domain analysis for the wavelet filtered data) is separately computed as well. MD: moderate disgusting; MF: moderate fearful; ED: extreme disgusting; EF: extreme fearful; ND: Neutral disgusting; NF: neutral fearful. 
bioRxiv preprint doi: https://doi.org/10.1101/2021.03.10.434892; this version posted April 20, 2021. The copyright holder for this preprint (which

was not certified by peer review) is the author/funder, who has granted bioRxiv a license to display the preprint in perpetuity. It is made available under aCC-BY-NC 4.0 International license.

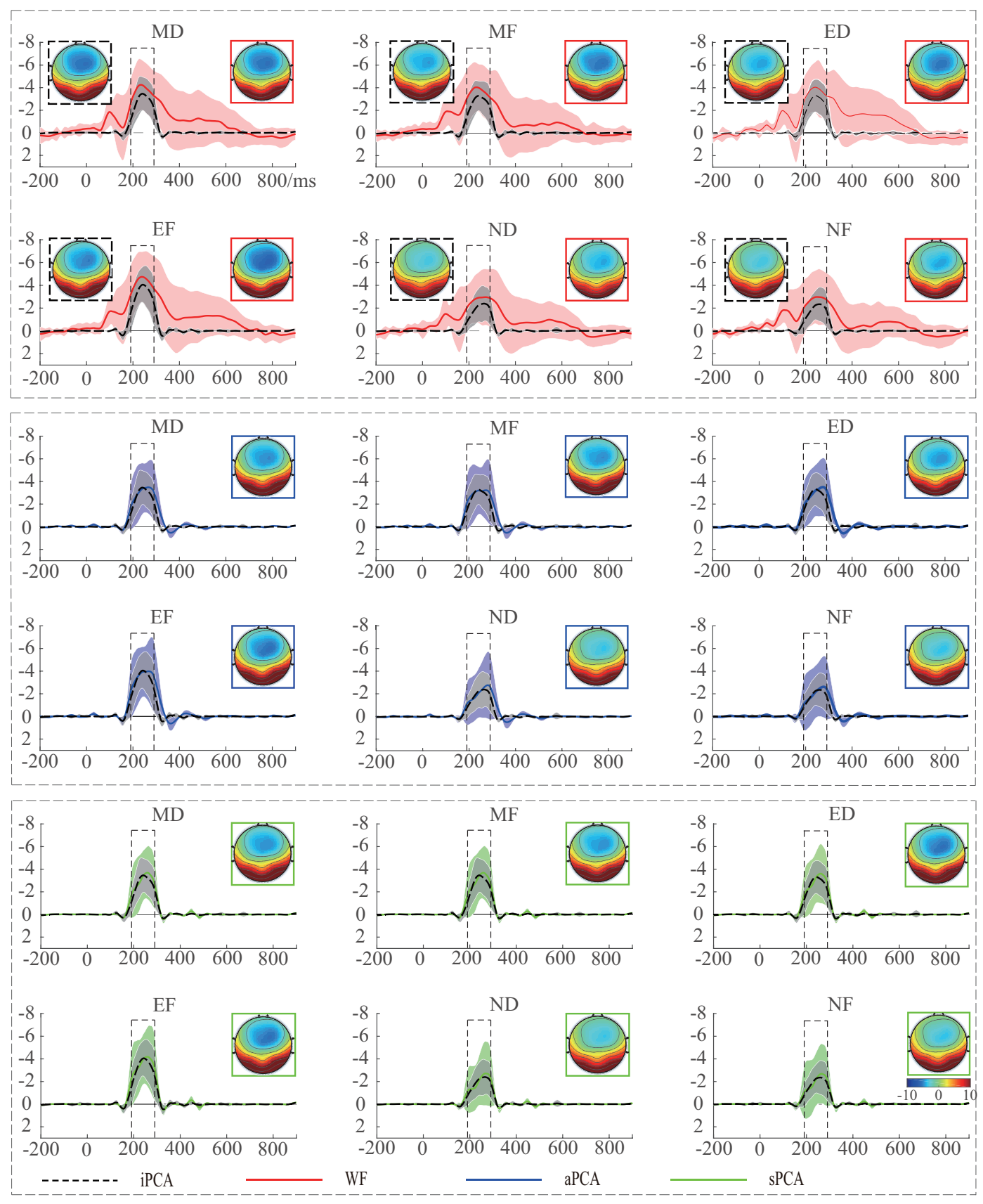

Figure 4: Grand averaged waveforms and topographies (mean measurement: from 190ms to $290 \mathrm{~ms}$ ) of different experimental conditions at $\mathrm{FCz}$ using WF (Upper panel: red solid line), iPCA (Upper panel: black dotted line), aPCA (Middle panel: blue solid line), and sPCA (Lower panel: green solid line). MD: moderate disgusting; MF: moderate fearful; ED: extreme disgusting; EF: extreme fearful; ND:29eutral disgusting; NF: neutral fearful. 
(a)
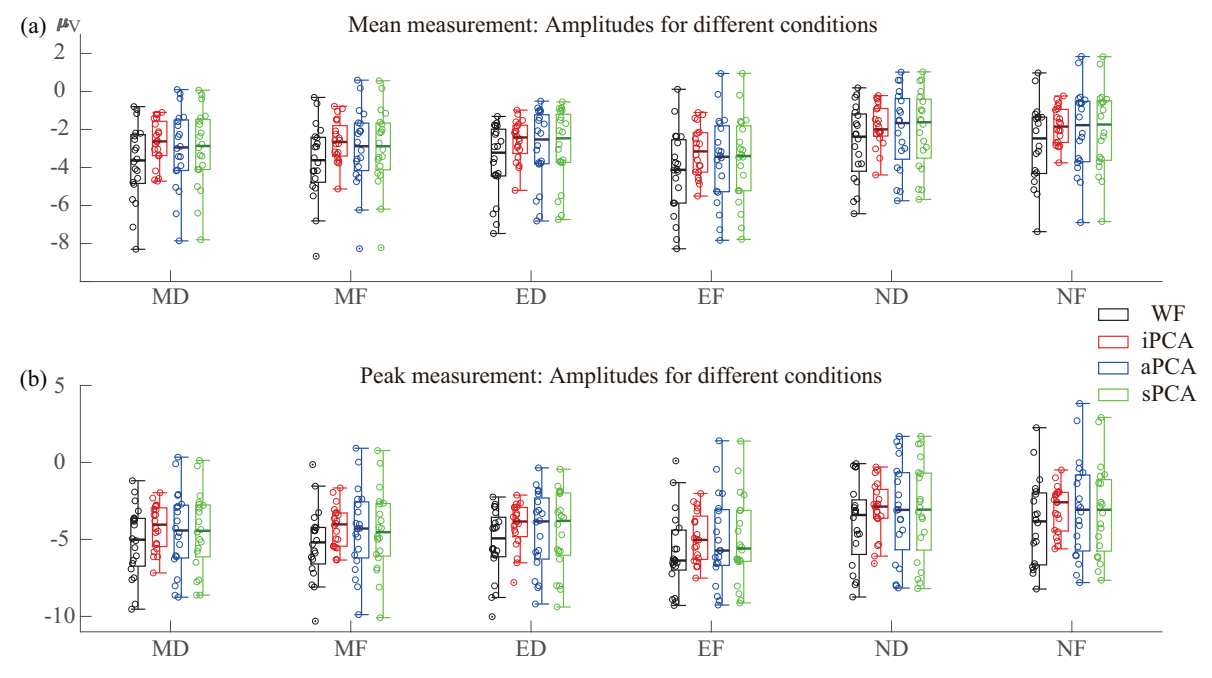

Figure 5: (a) and (b) are the peak/mean amplitudes of individuals (circles with different colours and time-window from 190 to $290 \mathrm{~ms}$ ) under different experimental conditions when using different techniques (WF, iPCA, aPCA, and sPCA), respectively. Noted that the standard value under each condition for the proposed technique is smaller than that of other techniques. MD: moderate disgusting; MF: moderate fearful; ED: extreme disgusting; EF: extreme fearful; ND: Neutral disgusting; NF: neutral fearful. 
(a) Mean measurement:The similarities of topographies beteen any two different subjects

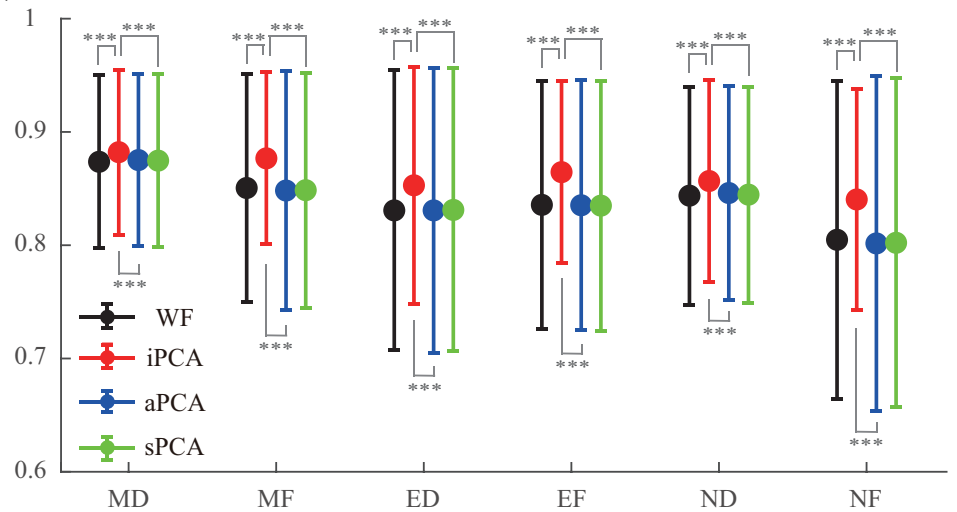

(b) Peak measurement:The similarities of topographies between any two different subjects

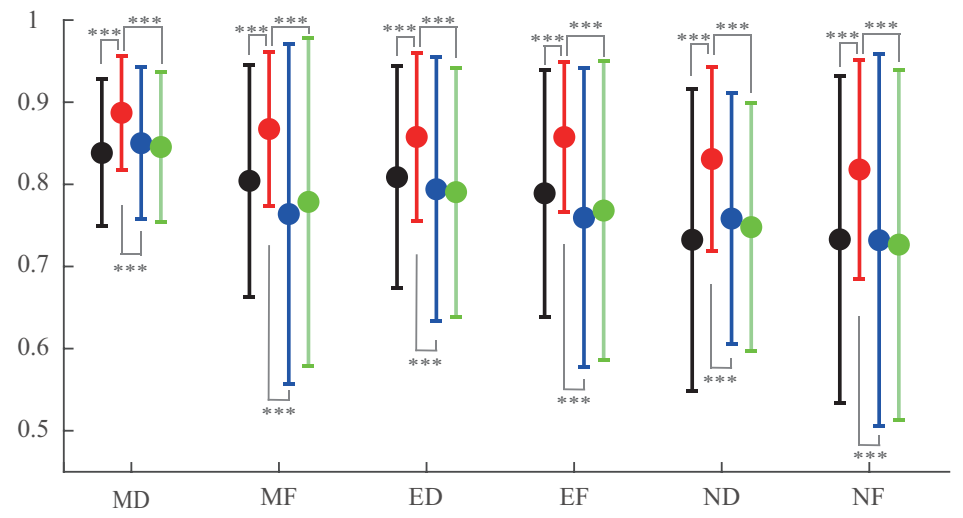

Figure 6: (a) and (b) correspond to the mean/standard values of similarities of topographies between any two different subjects under different experimental conditions for different techniques when using mean and peak measurements, respectively. The difference of similarities under each condition between the proposed technique and ant one of the other comparison techniques is confirmed by Paired t-test for mean and peak measurements separately $(* * * \mathrm{p}$ ¡0.001). MD: moderate disgusting; MF: moderate fearful; ND: extreme disgusting; EF: extreme fearful; ND: Neutral disgusting; NF: neutral fearful. 


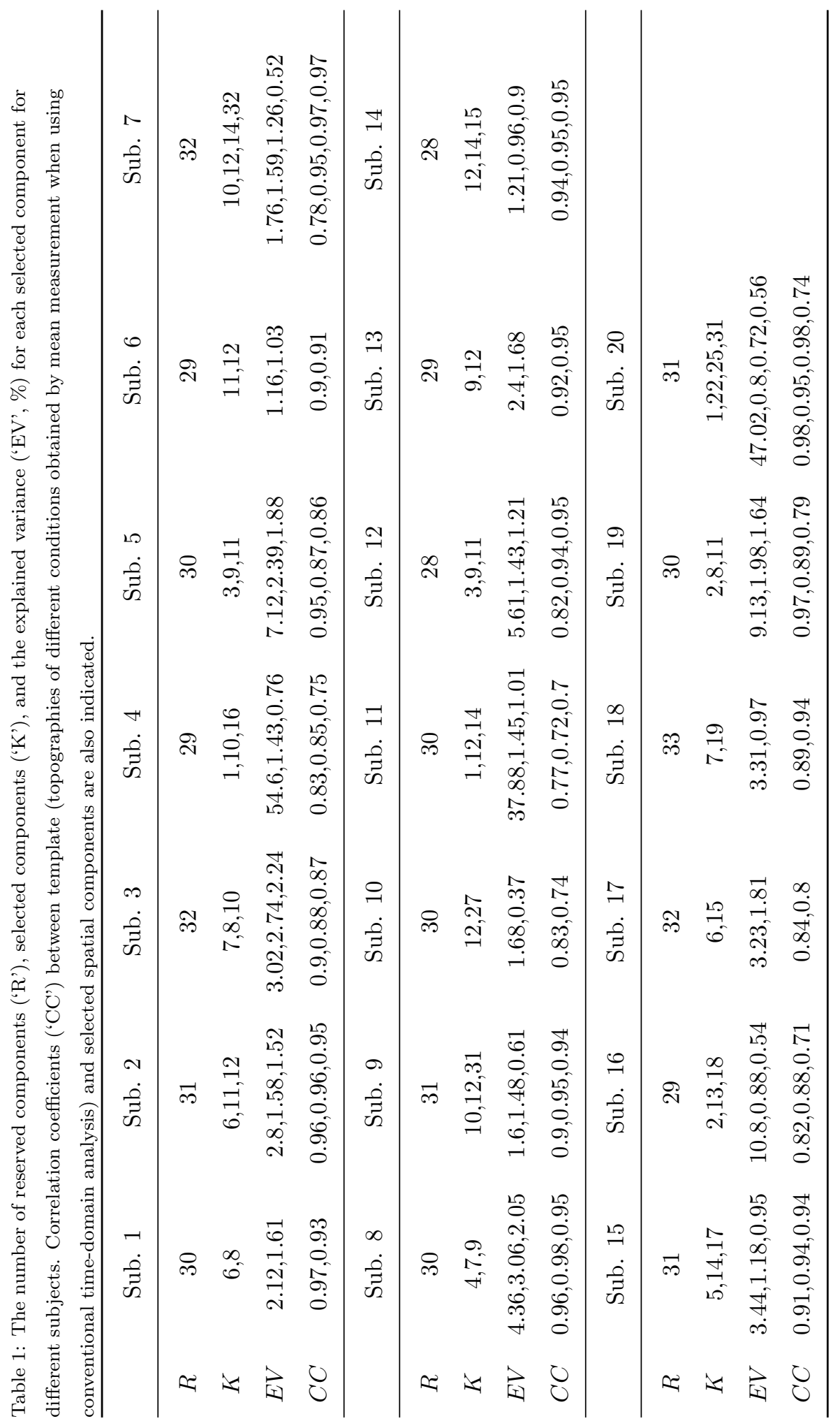

